\title{
Investigation of quadrupole deformation of nucleus and its surface dynamic vibrations
}

\author{
I. Boboshin ${ }^{1}$, B. Ishkhanov ${ }^{2}$, S. Komarov ${ }^{1}$, V. Orlin ${ }^{1}$, N. Peskov ${ }^{1}$, and V. Varlamov ${ }^{1, a}$ \\ Skobeltsyn Institute of Nuclear Physics of Lomonosov Moscow State University, 119992 Moscow, Russia \\ 2 Physics Faculty of Lomonosov Moscow State University, 119992 Moscow, Russia
}

\begin{abstract}
The new complete database - Chart of nuclear quadrupole deformations - was developed using various sources of related information. Using this Chart the system comparative analysis of quadrupole deformation parameters $\beta_{2}$ obtained by two different methods was carried out for many nuclei. The clear systematical disagreements of $\beta_{2}$ parameters obtained from nuclear quadrupole moments $\mathrm{Q}$ ("Q-type" data) and from reduced transition probability $\mathrm{B}(\mathrm{E} 2) \uparrow$ for $0^{+} \rightarrow 2_{1}^{+}$transitions ("B-type" data) were revealed. It was found out that all nuclides investigated are clearly separated into two groups. For all isotopes of "group 1" nuclei good agreement was observed for data of both types. For all isotopes of "group 2" nuclei "B-type" $\beta_{2}$ data values are systematically (in many cases significantly) larger than "Q-type" ones. In cases of only few exceptions disagreement for some isotopes are combined with agreements for others. It was shown that two types data difference could be explained in the frame of assumption about the not negligible role of dynamic vibrations of nucleus surface, by other words, about dependence of quadrupole deformation parameter $\beta_{2}$ values on surface vibrations of nucleus in ground state. From this point of view "Q-type" $\beta_{2}$ values do not take into account nucleus surface vibration, but "B-type" $\beta_{2}$ values do that. Therefore "B-type" data reflect not only static nuclear deformation (deviation of nucleus shape from spherical), but dynamic deformation also.
\end{abstract}

\section{Introduction}

Atomic nucleus matter space distribution is one of the most important fundamental physical features to which nuclear physics has a first priority interest. At last time amount of experimental researches devoted to studying nuclei shapes is increasing permanently, new experimental methods are developed, large volume nuclear structure parameters compilations are created. All those give to one possibility to obtain new data on various, first of all, quadrupole nuclear deformations.

Among the well-known systematical collections of data concerning quadrupole deformations, two could be pointed out as more complete and carefully obtained. The first one [1] is the experimental electrical quadrupole moment $\mathrm{Q}$ data collection including more than 1300 data for about 450 nuclides, continuing some previous collections [2,3]. The second one [4] is the collection of reduced transition probability $\mathrm{B}(\mathrm{E} 2) \uparrow$ values for $0^{+} \rightarrow 2_{1}^{+}$transitions (about 2000 $\mathrm{B}(\mathrm{E} 2) \uparrow$ values from $\sim 200$ publications for $\sim 1500$ nuclides) also continuing and adding previous collections.

At the Russia MSU SINP Centre for Photonuclear Experiments Data (CDFE) large amount of the quadrupole deformation parameter $\beta_{2}$ data from both collections mentioned have been used for development of the new database [5] - Chart of nuclear quadrupole deformations (http://cdfe.sinp.msu.ru/services/nsr/defchart/defmain.html).

The database is realized as a system analogue to wellknown Chart of nuclides (various properties of nuclides are sorted in $\mathrm{Z}$ and $\mathrm{N}$ coordinates). The colours of the Chart individual nuclide elements give the possibility for a comfortable overview of nuclei shapes and of finding nuclei having definite (speric, prolate or oblate) shape. Similar

${ }^{a}$ Presenting author, e-mail: Varlamov@depni .sinp.msu.ru to colours using for geographical maps preparation, brown colour was used for "hills" $\left(\beta_{2}>0\right)$, blue for "sears" $\left(\beta_{2}<0\right)$ and green for "plains" (unknown $\beta_{2}$ sign). Intensity of colour corresponds to the parameter absolute value.

Using new Chart the systematical analysis of $\beta_{2}$ parameters from various sources was carried out for many nuclei and at the first time revealed clear and in many cases significant systematical disagreements between quadrupole deformation parameters obtained using the two main methods mentioned.

Such complete databases are powerful tools for system analysis and overview of unknown agreements or, vice versa, disagreements of data obtained by various ways or for founding out new nuclear objects and investigation their new features. As an example of such kind research investigation of consistency between total and partial photonuclear reaction cross sections studied using different methods at various laboratories was carried out [6] at the MSU SINP CDFE using complete nuclear reaction relational database (http://cdfe.sinp.msu.ru/exfor/index.php). Several new (not classic traditional) magic nuclei have been found out and many their features investigated [7] using the possibilities of another complete nuclear structure database (http://cdfe.sinp.msu.ru/services/ensdfr.html).

\section{Two main methods for nucleus quadrupole deformation parameter obtaining}

\subsection{Quadrupole deformation parameter $\delta$ from nucleus quadrupole moment}

As is well known (for example, see [8]) the intrinsic quadrupole moment of evenly charged ellipsoid can be described 
by the equation

$$
Q_{0}=2 / 5 Z\left(b^{2}-a^{2}\right),
$$

where $a$ and $b$ are the small and large ellipsoid axises. Since quadrupole deformation parameter $\delta$ (degree of nucleus shape deviation from sphere) can be written as

$$
\delta=0.3\left(b^{2}-a^{2}\right) / 2\langle r\rangle^{2},
$$

where

$$
\left\langle r^{2}\right\rangle=\left(b^{2}+2 a^{2}\right) / 5,
$$

equation (1) would be written as follows

$$
Q_{0}=4 / 3 Z\left\langle r^{2}\right\rangle \delta
$$

Hence using experimental quadrupole moment $Q_{0}$ values [1] the nucleus quadrupole deformation parameter values $\delta$ could be calculated by the formula

$$
\delta=0.75 Q_{0} /\left(Z\left\langle r^{2}\right\rangle\right)
$$

Intrinsic moment $Q_{0}$ values were recalculated [9] from static electric quadrupole moment $Q$ values measured in the laboratory system. It was supposed that near spherical nucleus shape vibrations amplitude is small in comparison to nucleus equilibrium deformation and, therefore, the following equation can be used

$$
Q=Q_{0}\left(3 K^{2}-I(I+1)\right) /((I+1)(2 I+3)),
$$

where $I$ is the spin of the state - the member of rotational band based on nucleus ground state, $K$ is $I$ 's projection on symmetry axis.

Value of $\left\langle r^{2}\right\rangle$ was evaluated using the following expressions

$$
\begin{gathered}
\left\langle r^{2}\right\rangle=0.6 R_{0}^{2}\left(1+10 / 3\left(\pi a_{0} / R_{0}\right)^{2}\right) /\left(1+\left(\pi a_{0} / R_{0}\right)^{2}\right)(\mathrm{A} \leq 100), \\
\left\langle r^{2}\right\rangle=0.6\left(1.2 A^{1 / 3}\right)^{2}(\mathrm{~A}>100),
\end{gathered}
$$

which takes into account effects of light nuclei surface diffusion properties.

Parameters of radial Woods-Saxon potential form-factor $\left(R_{0}=1.07 A^{1 / 3} \mathrm{fm}\right.$ and $\left.a_{0}=0.55 \mathrm{fm}\right)$ were obtained from the data on fast electrons scattering.

\subsection{Quadrupole deformation parameter $\beta_{2}$ from reduced transition probability $\mathrm{B}(\mathrm{E} 2) \uparrow$ for $\mathrm{0}^{+} \rightarrow 2_{1}^{+}$ transition}

In [4] quadrupole deformation parameter $\beta_{2}$ was obtained using the following equation

$$
\beta_{2}=\left(4 \pi / 3 Z R_{0}^{2}\right)\left[B\left(E 2 ; 0^{+} \rightarrow 2_{1}^{+}\right) / e^{2}\right]^{1 / 2},
$$

where $B\left(E 2 ; 0^{+} \rightarrow 2_{1}^{+}\right)$is reduced probability for E2- transition $0^{+} \rightarrow 2_{1}^{+}$and $R_{0}^{2}=\left(1.2 A^{1 / 3} \mathrm{~cm}\right)^{2}$.

The values under discussion $\delta(5)$ and $\beta_{2}(9)$ are connected (for example, see [9]) as $\delta \approx 0.95 \beta_{2}$ and therefore further for simplicity the only symbol $\beta_{2}$ will be used.

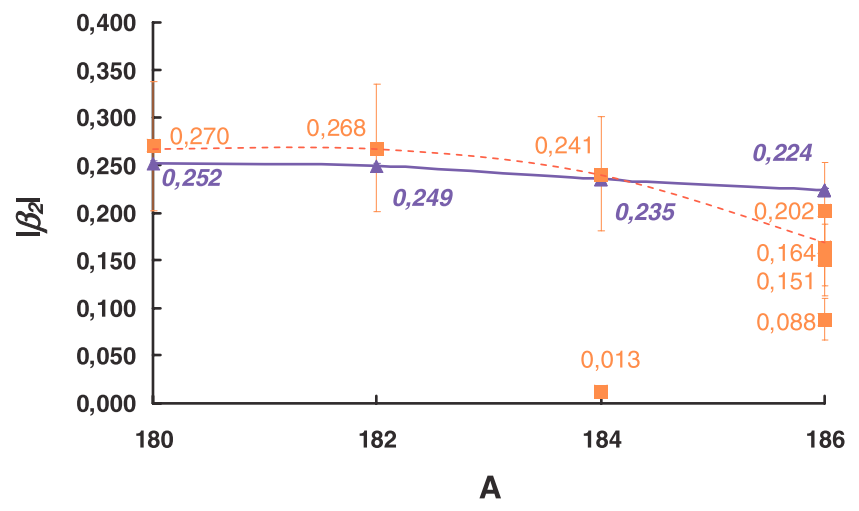

Fig. 1. Comparison of $\left|\beta_{2}\right|$ values obtained from $B(E 2) \uparrow$ data (blue triangles, solid line) and from $\mathrm{Q}$ data (red squares, dotted line) for $\mathrm{W}$ ("group 1").

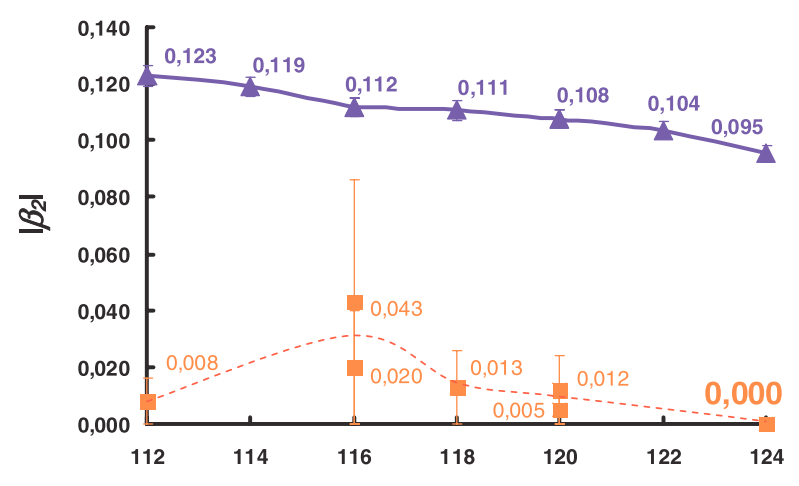

A

Fig. 2. Comparison of $\left|\beta_{2}\right|$ values obtained from B(E2) $\uparrow$ data and from Q data (designations are the same as in fig. 1) for Sn ("group $2 ") .{ }^{124}$ Sn case $\left(\left|\beta_{2}\right|_{Q}=0\right)$ is indicated specially.

\section{Comparison of data on quadrupole deformation parameter data obtained using two methods}

All $\left|\beta_{2}\right|$ values, obtained using two different methods described above ("Q-type" data from experimental quadrupole moments $\mathrm{Q}$ and "B-type" data from reduced transition probabilities $\mathrm{B}(\mathrm{E} 2) \uparrow)$ were investigated for many nuclei. It was found out that all nuclei studied are clearly separated into two groups ("1" and "2"):

- "group 1": for all isotopes of nuclei from "group 1" ( $\mathrm{Ti}, \mathrm{Cr}$, $\mathrm{Zr}, \mathrm{Nd}, \mathrm{Sm}, \mathrm{Gd}, \mathrm{Dy}, \mathrm{Er}, \mathrm{W}, \mathrm{Os}, \mathrm{Ra})$ "B-type" and "Q-type" data are very close to each other (small differences are of order of statistical uncertainties); the typical example for $\mathrm{W}$ isotopes is presented in figure 1.

- "group 2": for all isotopes of nuclei from "group 2" (C, Si, $\mathrm{Ar}, \mathrm{Ca}, \mathrm{Fe}, \mathrm{Ni}, \mathrm{Zn}, \mathrm{Ge}, \mathrm{Se}, \mathrm{Kr}, \mathrm{Sr}, \mathrm{Mo}, \mathrm{Ru}, \mathrm{Pd}, \mathrm{Cd}, \mathrm{Sn}$, $\mathrm{Te}, \mathrm{Ba}, \mathrm{Yb}, \mathrm{Hf}, \mathrm{Pt}, \mathrm{Pb}$ ) "B-type" data have values clearly larger than those for "Q-type" data; the typical example of comparison of both type data for $\mathrm{Sn}$ isotopes is presented in figure 2.

There are only few exceptions from the clear systematic obtained. It was founded out that in the cases of nuclei $\mathrm{Mg}$, $\mathrm{Xe}, \mathrm{Hg}$ and $\mathrm{U}$ phenomenon described is dependent on number 
of neutrons. For several isotopes "'B-type" data have values larger than those for "Q-type" data, but for others - vice versa, "Q-type" data have values larger than those for "B-type" data.

\section{Quadrupole deformation of nucleus and its surface vibration}

The difference between absolute value $\left|\beta_{2}\right|$ data derived from $\mathrm{Q}$ and $\mathrm{B}(\mathrm{E} 2) \uparrow$ values founded out can be explained in the fame of assumption about the connection of quadrupole deformation parameter with vibration of surface of nucleus in ground state. That is based on assumption that "Q-type" data do not take into account such nucleus surface vibrations, but "B-type" data do that. In other words from that point of view "B-type" data are affected not only by static nucleus deformation depended on its shape, but also by dynamic nucleus deformation that came from its surface vibration.

\subsection{Rotating nucleus model}

In the frame of rotating nucleus model it is supposed that rigid nucleus has a static deformation and its excitation is the result of nucleus rotating. It was shown [8] that value of reduced probability $\mathrm{B}(\mathrm{E} 2) \uparrow$ of transition between any states of rotating band can be expressed as

$$
B\left(E 2 ; K J_{1} \rightarrow K J_{2}\right)=(5 / 16 \pi) e^{2} Q_{0}^{2}\left\langle J_{1} K 20 \mid J_{2} K\right\rangle^{2},
$$

where $J$ is state spin, $K$ is spin projection to the axis of symmetry, $Q_{0}$ is intrinsic nucleus quadrupole moment, $\left\langle J_{1} K 20 \mid J_{2} K\right\rangle$ is vector summation coefficient. Partially, for E2 transition $0^{+} \rightarrow 2_{1}^{+}$it looks as

$$
B\left(E 2 ; 0^{+} \rightarrow 2^{+}\right)=\left((3 / 4 \pi) e Z R_{0}^{2}\right)^{2}\left(\beta_{2}\right)^{2}
$$

and completely corresponds to the expression (9).

\subsection{Vibrating nucleus model}

Because nucleus is not absolutely rigid (tough) object, its surface vibrates and amplitude of such vibrations depends on both excitation state energy and nucleus rigidity (toughness). It is obvious that if such vibrations occur the nucleus shape is changing. It is important that such vibrations can occur not only in excited states but in ground state also. Changing of nucleus shape (shape vibrations) occurs relative some "equilibrium" shape that can be partially spherical as in case of magic nuclei. It is important to underline that in such enough simple cases though in average nucleus shape is sphere, at different times initial photons are interacted with nuclei of different shapes. It is important that independently of the sign of deformation - for prolate or oblate nucleus - at the same absolute deformation parameter value interaction probability is the same. Both processes contributions are summed and therefore nucleus that is spherical in average can have not zero probability for transition $0^{+} \rightarrow 2_{1}^{+}$and hence not zero deformation parameter.
For simplicity: in an harmonic oscillator with spherical equilibrium shape, the average amplitude of nucleus shape difference from spherical one in ground state is

$$
\left\langle 0\left|\alpha_{2 \mu}\right| 0\right\rangle=0,
$$

where $\alpha_{v \mu}$ are collective variables describing nucleus surface motion, and $v=2$ for harmonic oscillator (that is true [9] for $\mathrm{N}$-th excited vibration state).

Squared deformation parameter $\beta_{0}^{2}$, characterized vibrating nucleus is defined [8] as average of the sum $\Sigma\left|\alpha_{2 \mu}\right|^{2}$

$$
\beta_{0}^{2}=\frac{\hbar}{2 B_{2} \omega_{2}}(5+2 N),
$$

where $B_{2}$ is mass coefficient, $\omega_{2}$ is vibration frequency and $N$ is phonon number.

It must be underlined that for nucleus ground state $(N=0)$

$$
\beta_{0}^{2}=\frac{5 \hbar}{2 B_{2} \omega_{2}} \neq 0(!)
$$

This phenomenon could have very simple explanation: dynamic deformation of nucleus in ground state occurs ((9), (11)) because not deviation of nucleus shape from "equilibrium" is averaged, but deviation squared. It must be underlined that both formulae $((9)$ and (11)) though being very similar (near identical) were obtained in quite different models and describe different physical processes taking place in nucleus at of $2^{+}$state excitation. The theory that could simultaneously describe both kind motions (vibration and rotation) of nucleus is very complicated and really in essence has not been developed. But at the same time it must be pointed out that in vibration model probability $B(E 2) \uparrow$ of transition $0^{+} \rightarrow 2_{1}^{+}$is connected [8] with introduced squared deviation $\beta_{0}^{2}$ of nucleus shape from equilibrium as

$$
B\left(E 2 ; n_{2}=0 \rightarrow n_{2}=1\right)=\left((3 / 4 \pi) e Z R_{0}^{2}\right)^{2}\left(\beta_{0}\right)^{2} .
$$

This expression textually (letter-to-letter) coincides to the expression (11). That gives to one possibility to interpret introduced $\beta_{0}$ as "deformation" also but namely "dynamic deformation" - not traditional "static" one.

It is obvious that the only simplest case could be analyzed. In reality vibrations occur relative not-spherical equilibrium shape and therefore much more complicated interaction of two (or more) types of moving must take place. As a result relatively simple and strict solution can not be obtained. But it is obvious nevertheless that effective interaction of two degrees of freedom will lead to effective increasing of deformation.

\section{Nucleus dynamic deformation}

The physical explanation of the phenomenon founded out can be the following. For "group 1" ("B-type" data are near to "Q-type" data) $2_{1}^{+}$- level excitation is pure rotational: vibration of nucleus in ground state ("zero vibration") is weak and the nucleus shape can be treated as "static". For "group 2" ("Btype" data are larger than "Q-type" ones) "zero vibrations" play important role. The superposition of static and dynamic deformations leads to effective increasing of nucleus deformation. As a matter of fact in this case the rotational model 
Table 1. Parameters characterised nucleus surface vibrations (" $C$ " is rigidity parameter, " $a$ " is vibration amplitude).

\begin{tabular}{llllllll}
\hline Nucl & $-\left.\beta_{2}\right|_{B}$ & $\left.\beta_{2}\right|_{Q}$ & $C_{2}$, & $C_{2 \beta}$, & $C_{2 \gamma}$, & $a_{\beta}$ & $a_{\gamma}$ \\
\hline${ }^{16} \mathrm{O}$ & 0.36 & $\approx 0.0$ & 132 & - & - & - & - \\
${ }^{18} \mathrm{O}$ & 0.36 & 0.09 & 39 & - & - & - & - \\
${ }^{24} \mathrm{Mg}$ & 0.61 & 0.44 & - & 82 & 36 & 0.20 & 0.24 \\
${ }^{28} \mathrm{Si}$ & 0.41 & 0.35 & - & 84 & 185 & 0.17 & 0.14 \\
${ }^{30} \mathrm{Si}$ & 0.32 & 0.09 & 56 & - & - & - & - \\
${ }^{40} \mathrm{Ca}$ & 0.12 & $\approx 0.0$ & 655 & - & - & - & - \\
${ }^{42} \mathrm{Ca}$ & 0.25 & 0.21 & - & 37 & 63 & 0.16 & 0.14 \\
${ }^{124} \mathrm{Sn}$ & 0.09 & $\approx 0.0$ & 293 & & & & \\
${ }^{144} \mathrm{Nd}$ & 0.13 & $\approx 0.0$ & 102 & - & - & - & - \\
${ }^{154} \mathrm{Sm}$ & 0.34 & 0.32 & - & 13 & 15 & 0.21 & 0.20 \\
${ }^{168} \mathrm{Er}$ & 0.34 & 0.34 & - & 16 & 7 & 0.19 & 0.24 \\
${ }^{178} \mathrm{Hf}$ & 0.28 & 0.27 & - & 20 & 19 & 0.17 & 0.18 \\
${ }^{182} \mathrm{~W}$ & 0.25 & 0.27 & - & 210 & 240 & 0.05 & 0.05 \\
${ }^{186} \mathrm{Os}$ & 0.20 & 0.20 & - & 205 & 107 & 0.05 & 0.06 \\
\hline
\end{tabular}

can not be employed. But because the formulae connected $\beta_{2}$ and $\mathrm{B}(\mathrm{E} 2) \uparrow$ are formally the same in both rotating (11) and vibrating (15) nucleus models it can be supposed that the "B-type" parameter of deformation is reflecting the effective increasing of nucleus deformation and such nucleus can be treated as trembled.

Several isotopes $\left({ }^{16} \mathrm{O},{ }^{40} \mathrm{Ca},{ }^{94} \mathrm{Mo},{ }^{96} \mathrm{Ru},{ }^{124} \mathrm{Sn}\right.$ (fig. 2), ${ }^{144} \mathrm{Nd}$ and some others) were founded out for which "Q-type" parameter of deformation $\left|\beta_{2}\right|_{Q} \approx 0$, but "B-type" one $\left|\beta_{2}\right|_{B}$ is large. The vibrating nucleus model in pure version can be applied for such isotopes and therefore $C_{2}$, spherical nucleus surface rigidity parameter, could be calculated (table):

$$
\left(\beta_{2}\right)_{B}^{2}=5 \hbar /\left(2 B_{2} \omega_{2}\right), \quad \hbar \omega_{2}=E_{2_{1}^{+}}=\hbar \sqrt{C_{2} / B_{2}} .
$$

For some other nuclei with relatively large static deformation and relatively weak $\beta$ - and $\gamma$-vibrations rotational lines could be looked through and correspondingly deformed nucleus rigidity parameters $C_{2 \beta}, C_{2 \gamma}$ and vibration amplitudes $a_{\beta}, a_{\gamma}$ could be calculated (if correspondent formulae could work in conditions under discussion):

$$
\begin{gathered}
\hbar \omega_{\beta}=E_{0_{1}^{+}}=\hbar \sqrt{C_{2 \beta} / B_{2}}, \quad \hbar \omega_{\gamma}=E_{2_{1}^{+}}=\hbar \sqrt{C_{2 \gamma} / B_{2}} \\
E_{2_{1}^{+}}=\hbar^{2} J(J+1) /\left.\left(6 B_{2} \beta_{2}^{2}\right)\right|_{J=2}=\hbar^{2} /\left(B_{2} \beta_{2}^{2}\right), \\
a_{\beta, \gamma}^{2}=\hbar /\left(2 B_{2} \omega_{\beta, \gamma}\right) .
\end{gathered}
$$

One can see that isotopes ${ }^{16} \mathrm{O},{ }^{40} \mathrm{Ca},{ }^{124} \mathrm{Sn}$, and ${ }^{144} \mathrm{Nd}$ have near zero static deformation but clear dynamic one. It is interesting that isotope ${ }^{40} \mathrm{Ca}$ (double magic spherical nucleus) is very rigid ( $C_{2}$ is large) but nevertheless its surface vibrates: clear dynamic deformation. Therefore it could be treated as "trembling" in ground state. Vice versa, ${ }^{42} \mathrm{Ca}$ is deformed and soft (both $C_{2}$ are relatively small) nucleus, but its surface does not vibrate and therefore its deformation is clear "static".

So research carried out leads to conclusions that: there are many nuclei for all isotopes of which both-type $\beta_{2}$ data are of the same value; those nuclei could be spherical or deformed but their surfaces do not vibrate, there are many nuclei for all isotopes of which "B-type" $\beta_{2}$ data have values larger (in some cases significantly (factor $~ 1.5-2.0$ )) than "Q-type" data; the surfaces of such nuclei in ground states vibrate and therefore such nuclei have clear dynamic deformation and could be treated as "trembling", there are only few nuclei for which the intermediate situation is observed, there are absolutely no nuclei for which "Q-type" data are larger than "B-type" ones. So two types $\beta_{2}$ parameter data difference found out could be explained in the frame of assumption about dynamic surface vibrations of nucleus in ground state: "B-type" data reflect not only static nuclear deformation (deviation of nucleus shape from spherical), but dynamic deformation also.

Authors acknowledge very much Prof. N.J. Stone for presentation of complete nuclei quadrupole moments values collection digital data and Prof. Yu.P. Gangrsky for important ideas and fruitful discussions. Research was carried out at the MSU SINP DEPANI and supported by the President of Russia Grant N SS-1619.2003.2.

\section{References}

1. N.J. Stone, At. Data Nucl. Data Tables 90, 75 (2005).

2. N.J. Stone, Preprint Oxford Physics (Claredon Laboratory OXFORD OX1 3PU U.K., 2001).

3. P. Raghavan, At. Data Nucl. Data Tables 42, 189 (1989).

4. S. Raman, C.W. Nestor, P. Tikkanen, At. Data Nucl. Data Tables 78, 1 (2001).

5. I.N. Boboshin, V.V. Varlamov, S. Yu. Komarov et al., Proceedings of the International Conference Nuclear Structure and Related Topics, Dubna, Russia, 2006 (ISBN 5-9530-0113-4, JINR Dubna, 2006), p. 23.

6. I.N. Boboshin, B.S. Ishkhanov, V.V. Varlamov, Phys. At. Nuclei 67, 1846 (2004)

7. V.V. Varlamov, N.N. Peskov, D.S. Rudenko, M.E. Stepanov, INDC(CCP)-440, IAEA NDS, Vienna, Austria, 2004, p. 37.

8. I.M. Kapitonov, Introduction into Nuclei and Particles Physics (EDITORIAL URSS, Moscow, 2002).

9. A. Bohr, B.R. Mottelson, Structure of Atomic Nucleus (MIR, Moscow, 1971).

10. I. Heisenberg, V. Greiner, Nuclear Models. Collective and Single-Particle Phenomena (ATOMIZDAT, Moscow, 1975). 